\title{
Layer-Dependent Mechanical Properties and Enhanced Plasticity in the Van der Waals Chromium Trihalide Magnets
}

\author{
Fernando Cantos-Prieto, ${ }^{\dagger}$ Alexey Falin, ${ }^{\dagger}$ Martin Alliati, Dong Qian, Rui Zhang, Tao Tao, \\ Matthew R. Barnett, Elton J. G. Santos,* Lu Hua Li,* and Efrén Navarro-Moratalla*
}

Cite This: Nano Lett. 2021, 21, 3379-3385

Read Online

ABSTRACT: The mechanical properties of magnetic materials are instrumental for the development of magnetoelastic theories and the optimization of strain-modulated magnetic devices. In particular, twodimensional (2D) magnets hold promise to enlarge these concepts into the realm of low-dimensional physics and ultrathin devices. However, no experimental study on the intrinsic mechanical properties of the archetypal 2D magnet family of the chromium trihalides has thus far been performed. Here, we report the room temperature layer-dependent mechanical properties of atomically thin $\mathrm{CrCl}_{3}$ and $\mathrm{CrI}_{3}$, finding that the bilayers have Young's moduli of 62.1 and $43.4 \mathrm{GPa}$, highest sustained strains of $6.49 \%$ and $6.09 \%$ and breaking strengths of 3.6 and $2.2 \mathrm{GPa}$, respectively.
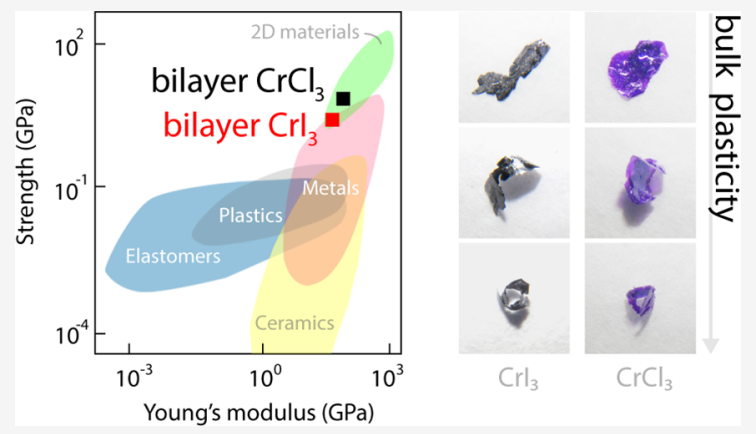
This portrays the outstanding plasticity of these materials that is qualitatively demonstrated in the bulk crystals. The current study will contribute to the applications of the $2 \mathrm{D}$ magnets in magnetostrictive and flexible devices.

KEYWORDS: 2D magnetic materials, mechanical properties, strain tunability, nanoindentation, Young's modulus, plasticity

$\mathrm{T}^{\mathrm{k}}$ he magnetic moment of a crystal is susceptible to the application of external strain, ${ }^{1}$ as a consequence magnetostriction has had a big technological relevance in the past $^{\text {century. }^{2-4}}$ The recent isolation of free-standing 2D magnets, ${ }^{5-9}$ has settled long-standing fundamental questions ${ }^{7}$ and enabled ultrathin magnetoelectric devices. ${ }^{10-12}$ However, despite recent works that have demonstrated a strong modulation of $2 \mathrm{D}$ magnetism in atomically thin $\mathrm{CrI}_{3}$ under high-pressure values, ${ }^{13,14}$ direct-strain modulation has only been attempted for $<0.3 \%$ strain values, ${ }^{15}$ and the prospects of the modulation of magnetism in the $2 \mathrm{D}$ limit have therefore not been fully explored. This can be attributed to a lack of fundamental understanding of the intrinsic mechanical properties of $2 \mathrm{D}$ magnets, which proves vital to realize their various applications. Indeed, although $\mathrm{CrCl}_{3}$ was first studied by Kamerlingh Onnes ${ }^{16}$ at the beginning of the last century, no experimental data on the mechanical properties of the magnetic chromium trihalide $\left(\mathrm{CrX}_{3}, \mathrm{X}=\mathrm{I}, \mathrm{Cl}, \mathrm{Br}\right)$ bulk or few-layer crystals has been reported to date.

The mechanical properties of $2 \mathrm{D}$ materials have been shown to be different from those of their bulk counterparts. Graphene, for instance, has Young's modulus of $\sim 1 \mathrm{TPa}$ and breaking strength of $130 \mathrm{GPa},{ }^{17}$ significantly higher than in graphite. ${ }^{18,19}$ A similar trend has been observed in other 2D materials, such as atomically thin hexagonal boron nitride (hBN) (0.87 TPa in Young's modulus and $70 \mathrm{GPa}$ in breaking strength) and molybdenum disulfide $\left(\mathrm{MoS}_{2}\right)(0.33 \mathrm{TPa}$ in
Young's modulus and $30 \mathrm{GPa}$ in breaking strength). ${ }^{20,21}$ It is worth mentioning that these strength values are far beyond the yield strength measured in conventional materials (i.e., $\sim 3 \mathrm{GPa}$ for that of silicon), ${ }^{22}$ demonstrating the capability of $2 \mathrm{D}$ materials to sustain an enormous strain without failure, ${ }^{23}$ for example, up to $25 \%$ in the case of graphene. ${ }^{17}$ On the other hand, the multilayer forms of these van der Waals crystals can benefit from their layered structure to achieve large plasticity. Such exceptional behavior has been recently reported in InSe, portraying this material as a strong candidate for near-future deformable electronics. ${ }^{24,25}$ It is therefore timely to explore the layer-dependent intrinsic mechanical properties of the chromium trihalides as archetypal magnetic $2 \mathrm{D}$ materials.

In our experiment, we obtained atomically thin $\mathrm{CrI}_{3}$ and $\mathrm{CrCl}_{3}$ flakes down to the bilayer (2L) by mechanical exfoliation of bulk crystals. The exfoliation was directly performed on substrates with prefabricated microwells for atomic force microscopy (AFM) nanoindentation (see Supporting Information for details). Figure 1a,d shows an optical micrograph of atomically thin $\mathrm{CrI}_{3}$ and $\mathrm{CrCl}_{3}$ covering

Received: December 4, 2020

Revised: March 29, 2021

Published: April 9, 2021 

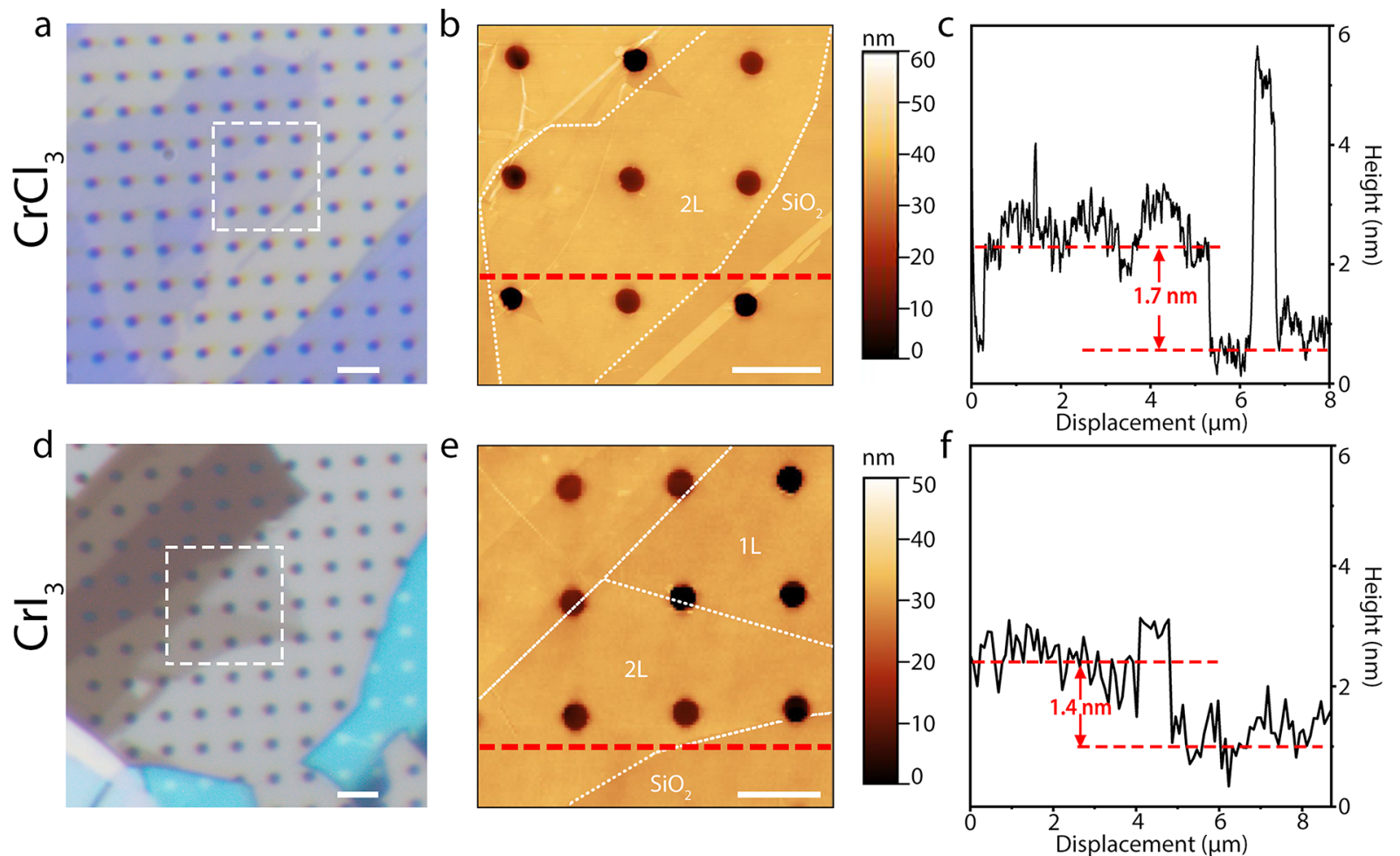

Figure 1. Characterization of $\mathrm{CrX}_{3}$ nanosheets. (a, d) Optical microscopy image of $2 \mathrm{~L}$ and few-layers of $\mathrm{CrCl}_{3}$ and $\mathrm{CrI}_{3}$ crystals, respectively, on a $\mathrm{SiO}_{2} / \mathrm{Si}$ substrate suspended over microwells of $600 \mathrm{~nm}$ in diameter; (b, e) AFM image of the $\mathrm{CrCl}_{3}$ and $\mathrm{CrI}_{3}$ thin crystals, respectively, corresponded to the square area of optical images ( $a, d$, respectively); and $(c, f)$ the corresponding height traces of the dashed line in panels $b$ and $e$ of $2 \mathrm{~L} \mathrm{CrCl}_{3}$ and $2 \mathrm{~L} \mathrm{CrI}_{3}$ crystals, respectively. Scale bars in white, $3 \mu \mathrm{m}$ in panels a and $\mathrm{d}$ and $2 \mu \mathrm{m}$ in panels b and e.
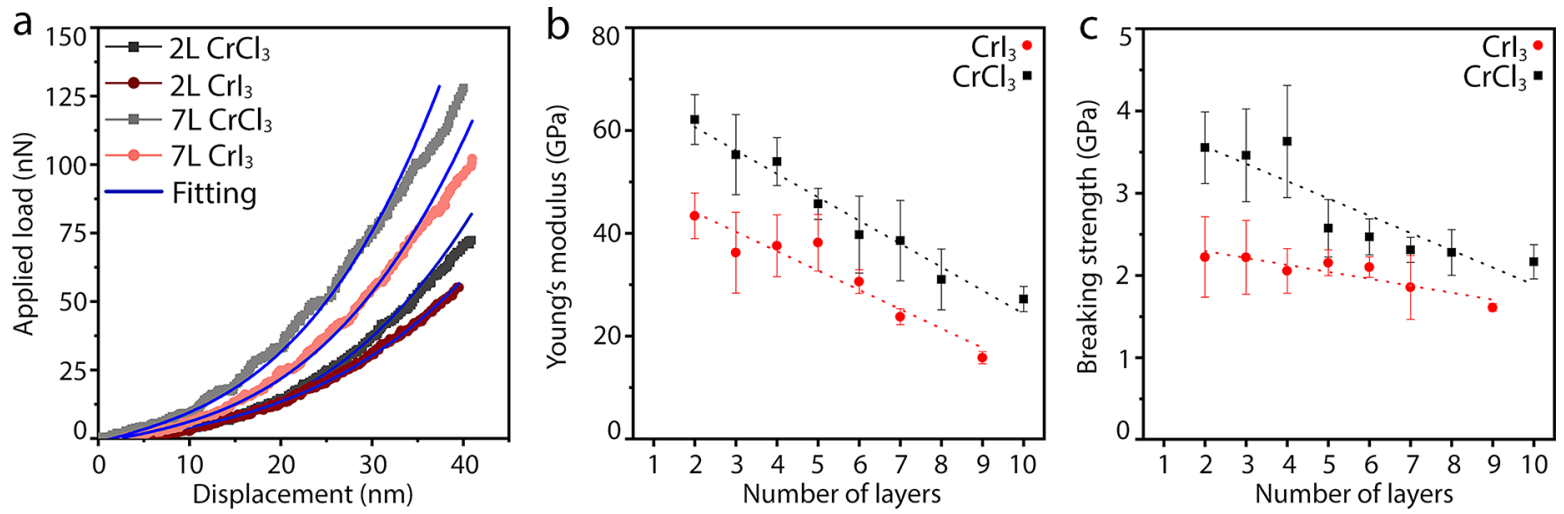

Figure 2. Mechanical properties of $\mathrm{CrX}_{3}$ nanosheets. (a) Load-displacement curves and the corresponding fittings for $2 \mathrm{~L}, 7 \mathrm{~L}$ for $\mathrm{CrCl}_{3}$ and $\mathrm{CrI}_{3}$; (b) volumetric Young's modulus; and (c) breaking strength of $\mathrm{CrCl}_{3}$ and $\mathrm{CrI}_{3}$ crystals of different thicknesses, along with dashed lines that show the linear fit of experimental Young's moduli and breaking strength values.

several holes in a $90 \mathrm{~nm}-\mathrm{SiO}_{2} / \mathrm{Si}$ substrate. Figure $\mathrm{lb}$ and $\mathrm{e}$ shows the corresponding AFM images in contact mode, portraying a thickness of 1.7 and $1.4 \mathrm{~nm}$ (Figure 1c,f), respectively, which corresponds to $2 \mathrm{~L} \mathrm{CrCl}_{3}$ and $\mathrm{CrI}_{3}$.

The mechanical properties of the few-layer $\mathrm{CrI}_{3}$ and $\mathrm{CrCl}_{3}$ were probed by the nanoindentation technique performed with the same AFM used for topographic inspection. ${ }^{17,20}$ The loaddisplacement curves were obtained by applying a load at the center of each suspended region until fracture for a minimum of five indentations per thickness per material to ensure the repeatability of the results. The curves were then fitted by a well-established model ${ }^{17}$ (see section 4 of the Supporting Information) as demonstrated in Figure 2a. From these results, we extracted Young's modulus $(E)$ for both materials in terms of their layer count (Figure 2b). The breaking strength $(\sigma)$ (Figure 2c) and ultimate strain values were determined based on the obtained fracture loads and the load-displacement relationships by means of finite element simulation (FEM) (see section 5 in the Supporting Information). The volumetric Young's modulus and breaking strength of $2 \mathrm{~L} \mathrm{CrI}_{3}$ and $2 \mathrm{~L}$ $\mathrm{CrCl}_{3}$ were $E=43.4 \pm 4.4 \mathrm{GPa}$ and $\sigma=2.2 \pm 0.5 \mathrm{GPa}$ and $E=$ $62.1 \pm 4.8 \mathrm{GPa}$ and $\sigma=3.6 \pm 0.4 \mathrm{GPa}$, respectively. The ultimate strain was found directly under the tip and its values for $2 \mathrm{~L} \mathrm{CrI}_{3}$ and $2 \mathrm{~L} \mathrm{CrCl}_{3}$ were $6.09 \%$ and $6.49 \%$, respectively. A direct comparison between the two materials indicates that both the Young's modulus and the breaking strength of $\mathrm{CrCl}_{3}$ were larger than those of $\mathrm{CrI}_{3}$, depicting that the chromium trihalide materials with a heavier halide exhibit a lower 

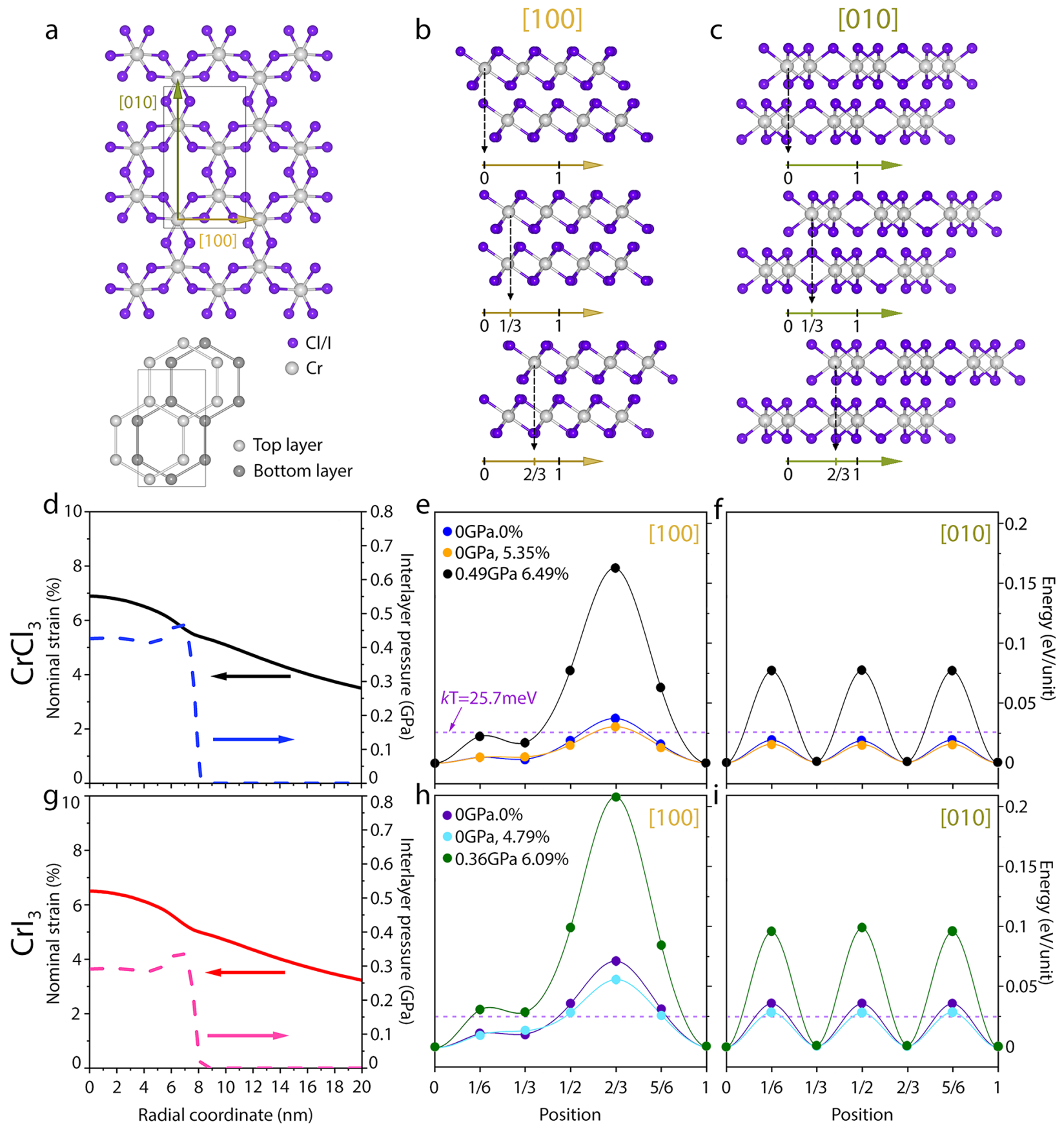

Figure 3. Sliding energies of bilayer $\mathrm{CrX}_{3}$ under different in-plane strain and out-of-plane compression conditions. (a) Top-view of the bilayer structure utilized in the vdW-DFT simulations, respectively. Two high-symmetry directions along the [100] and [010] are considered as representatives of the lateral sliding process occurring in the structures. Only $\mathrm{Cr}$ atoms are shown in a bottom to highlight the bilayer stacking. (b, c) Different positions correspond to fractional lateral shifts of the top-layer relative to the original $\mathrm{AB}$ stacking in units of $[1 / 6,0]$ and $[1 / 6,-1 / 6]$ over the unit cell along [100] and [010], respectively. (d) FEM calculations of nominal strain (solid line) and interlayer pressure (dashed line) in $2 \mathrm{~L} \mathrm{CrCl}_{3}$ within a radial distance of $20 \mathrm{~nm}$ from the indentation center, where three distinct regions were chosen to study sliding energy per formula unit (eV/unit) of bilayer $\mathrm{CrCl}_{3}$ along (e) [100] and (f) [010], respectively. The sliding energy was determined with the Cr atoms being fixed and the $\mathrm{Cl}$ atoms being relaxed in the simulation (see Figures S8-S10 for additional details). The dashed line indicates available thermalenergy at room temperature $(k T=25.7 \mathrm{meV})$. Colored dots are the calculated vdW-DFT energies with a cubic interpolation (solid lines) between different positions. $(\mathrm{g}-\mathrm{i})$ Analogous analysis as in panels $\mathrm{d}-\mathrm{f}$ for $2 \mathrm{~L} \mathrm{CrI}_{3}$. The monoclinic (space group $\mathrm{C} 2 / \mathrm{m}$ ) stacking order was utilized in all simulations.

mechanical stiffness. This trend correlates nicely with the ionic character of the $\mathrm{Cr}-\mathrm{X}$ bond, which is stronger in the $\mathrm{Cr}-\mathrm{Cl}$ interaction compared to $\mathrm{Cr}-\mathrm{I}^{26}$

Remarkably, as the thickness of the flakes increases, both atomically thin materials show a drop in Young's modulus and breaking strength. For example, $9 \mathrm{~L} \mathrm{CrI}_{3}$ had $E=15.8 \pm 1.2$
$\mathrm{GPa}$ and $\sigma=1.6 \pm 0.04 \mathrm{GPa}$, representing a $64 \%$ and $27 \%$ decrease in Young's modulus and breaking strength compared to $2 \mathrm{~L}$, respectively. Similar trends were observed in $\mathrm{CrCl}_{3}$, where $10 \mathrm{~L} \mathrm{CrCl}_{3}$ had $E=27.1 \pm 2.5 \mathrm{GPa}$ and $\sigma=2.2 \pm 0.2$ $\mathrm{GPa}$. To provide further insights into the layer-dependent mechanical properties of the chromium trihalides, we have 
undertaken van der Waals-corrected density functional theory (vdW-DFT) calculations to unveil the energy landscape of interlayer sliding shifts during the mechanical tests (see section 6 in the Supporting Information for details). Figure 3a shows a schematic of the atomic structure of bilayer $\mathrm{CrX}_{3}$ along the crystallographic $c$ axis (direction of the AFM tip motion during indentation) in the monoclinic phase (space group $\mathrm{C} 2 / \mathrm{m}$ ) present at room temperature, with the definition of the two interlayer sliding paths utilized in the simulations: along [100] and along [010]. We apply a fractional lateral shift on one chromium-trihalide layer relative to the other starting from the $\mathrm{AB}$ stacking order (Figure $3 \mathrm{~b}, \mathrm{c}$ ). The FEM simulations predict that the suspended $2 \mathrm{~L} \mathrm{CrX}$ crystals are mostly under small inplane strain in the area far from the contact region even under the fracture loads. This picture changes in the region close to the indentation center where out-of-plane compression starts to play a key role in the fracture mechanism. Figure $3 \mathrm{~d}$ and $3 \mathrm{~g}$ shows the in-plane strain (solid lines) and out-of-plane compression (dashed lines) distributions close to the indentation center under different fracture loads for $2 \mathrm{~L}$ $\mathrm{CrCl}_{3}$ and $2 \mathrm{~L} \mathrm{CrI}_{3}$, respectively. On the vdW-DFT calculations, three distinct regions were chosen to evaluate the sliding energy barriers. In the region far away from the indentation center, the equilibrium interlayer interaction occurs at $0 \mathrm{GPa}$ out-of-plane compression and $0 \%$ in-plane strain (i.e., $0 \mathrm{GPa}$ and $0 \%$ for both $\mathrm{CrCl}_{3}$ and $\mathrm{CrI}_{3}$ ). This choice of strain conditions is a valid approximation to our experiments, where extremely low values of strain, $<0.5 \%$, are found at the membrane edges (see Figure S3). The area just outside of the contact region is under a large in-plane strain but without any out-of-plane compression $(5.35 \%$ and $0 \mathrm{GPa}$ for $\mathrm{CrCl}_{3} ; 4.79 \%$ and $0 \mathrm{GPa}$ for $\mathrm{CrI}_{3}$ ). The tip contact region experiences the highest in-plane strain and out-of-plane compression under the fracture loads (0.49 GPa and $6.49 \%$ for $\mathrm{CrCl}_{3} ; 0.36 \mathrm{GPa}$ and $6.09 \%$ for $\mathrm{CrI}_{3}$ ). Figure $3 \mathrm{e}, 3 \mathrm{f}, 3 \mathrm{~h}$, and $3 \mathrm{i}$ summarizes the sliding energy per formula unit obtained for $\mathrm{CrI}_{3}$ and $\mathrm{CrCl}_{3}$ at different values of interlayer pressure and inplane strain as provided by FEM simulations. In the regions of the membranes beyond $\sim 8.5 \mathrm{~nm}$ from the indentation centers (see Table S2), where no pressure and small strain are present in the systems, the individual layers of $2 \mathrm{~L} \mathrm{CrI}_{3}$ and $\mathrm{CrCl}_{3}$ tend to slide over each other despite the path considered, that is, [100] or [010] (Figure $3 \mathrm{e}-\mathrm{f}$ and $3 \mathrm{~h}-\mathrm{i}$ ). This process is mediated by thermal fluctuations $(k T=25.7 \mathrm{meV})$, which are present at room temperature. The interlayer barriers are below $k T$ for the majority of the positions with the only exception at the fractional shift of $2 / 3$. At this crystallographic position, there is a slight increment of the energy above $k T$, which prevents further sliding along both [100] and [010]. This indicates that the layers can displace almost freely with little energetic opposition (Figure $3 \mathrm{f}$ and $3 \mathrm{i}$ ). As pressure and strain are applied (see Table S2), there is an increment of the energetic barriers at $2 / 3$ along [100] for $\mathrm{CrCl}_{3}(168 \mathrm{meV})$ and $\mathrm{CrI}_{3}(209 \mathrm{meV})$ which indicates that the layers may find difficulties to slide over at that particular position (Figure $3 \mathrm{e}$ and $3 \mathrm{~h}$ ). The main driving force for such enhancement of the energies is the strong overlap of the charge density at $2 / 3$ (Figure S5). Conversely, along [010] at $0.49 \mathrm{GPa}$ and $6.49 \%$, and $0.36 \mathrm{GPa}$ and $6.09 \%$ for both $\mathrm{CrCl}_{3}$ and $\mathrm{CrI}_{3}$, respectively, the energies at $2 / 3$ and their multiple positions $(0,1 / 3,1)$ are below kT (Figure $3 \mathrm{f}$ and $3 \mathrm{i}$ ) but slightly increments at intermediate positions $(1 / 6,1 / 2,5 / 6)$ although still smaller than the barrier at $2 / 3$ along $[100]$ at finite strain and pressure.
This suggests that the layers may choose a combination of sliding paths to minimize their energies as the pressure is applied. That is, the layers may start along the path [100] but may change direction to [010] to minimize their energies. Since the sliding path from $1 / 2$ to $2 / 3$ along [100] is symmetrically the same as along [010] (see Figure S7), the layers will follow a downhill energy profile from $\sim 76\left(\mathrm{CrCl}_{3}\right)$ and $\sim 98 \mathrm{meV}\left(\mathrm{CrI}_{3}\right)$ to $0 \mathrm{meV}$ on both cases rather than increase their energy following the same path along [100]. These results are consistent with the variation of mechanical properties versus the number of layers which follows our previous analysis on graphene and $\mathrm{hBN}^{20}$ providing a plausible explanation for the layer-dependence of the mechanical properties in $\mathrm{CrX}_{3}$. It is worth mentioning that the energetic barriers observed in the sliding of the layers are particularly sensitive to the relaxation of the atoms involved $(\mathrm{Cr}, \mathrm{Cl}, \mathrm{I})$ during the computation. Figure S8 shows results without any relaxation in the layers, which resulted in larger barriers. Indeed, lower energies than those shown in Figure $3 \mathrm{e}-\mathrm{f}$ and Figure $3 \mathrm{~h}-\mathrm{i}$ can be achieved when the relaxation of the $\mathrm{Cr}$ atoms is also taken into account (see Figure S9) with a consequent expansion of the interlayer distance (see Figure S10). This correlates well with the positions where the energies increase during the sliding and suggests that changes of stacking order should be followed by expansion or contraction of the interlayer distance as recently measured. ${ }^{14}$ In addition, the magnitudes of the barriers for $\mathrm{CrI}_{3}$ are moderately larger than those for $\mathrm{CrCl}_{3}$, which suggests a slightly more stable dependence of the mechanical properties with the thickness, in agreement with the overall experimental trend observed.

Overall, the measured mechanical values are among the smallest ones observed within the family of $2 \mathrm{D}$ materials, that is, much less stiff than $2 \mathrm{D}$ transition metal dichalcogenides and mica. $^{21,27,28}$ Figure 4 shows a map of the mechanical properties

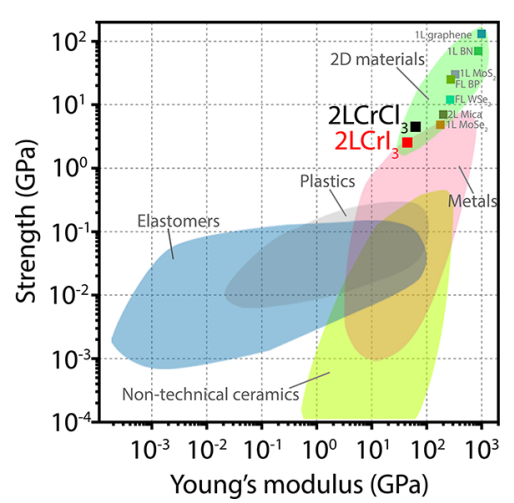

Figure 4. Map of mechanical properties of different materials in Young's modulus-Ultimate/breaking strength space. The mechanical properties of different types of materials, including $2 \mathrm{D}$ crystals and measured here $2 \mathrm{~L}$ of $\mathrm{CrX}_{3}$, are compared.

of atomically thin $\mathrm{CrX}_{3}$ compared to other materials. The position of $\mathrm{CrX}_{3}$ on this chart can be qualitatively explained by taking into account the bonding energies inside of the crystal, which scale according to the magnitudes of Young's modulus and breaking strength. While the dissociation energy for the honeycomb of $\mathrm{C}$ atoms in graphene yields a value of $805 \mathrm{~kJ} /$ $\mathrm{mol},{ }^{29}$ our DFT calculations indicate a formation energy of $260.9 \mathrm{~kJ} / \mathrm{mol}$ for $\mathrm{CrI}_{3}$ and $597.7 \mathrm{~kJ} / \mathrm{mol}$ for $\mathrm{CrCl}_{3}$, depicting a weaker interatomic interaction than that of the graphene lattice. In addition, within the chromium halide family, the 

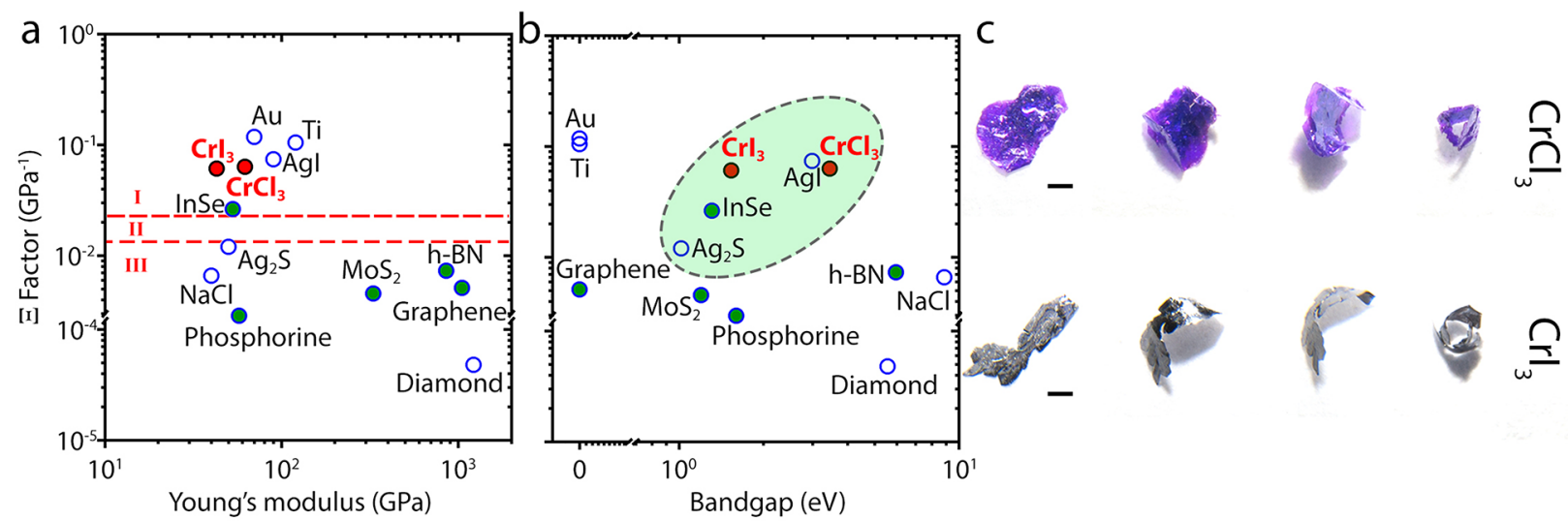

Figure 5. Deformability factor and enhanced plastic behavior of multilayer $\mathrm{CrX}_{3}$. (a) Deformability factor dependence on the Young Modulus, Regions I, II, and III correspond to plastic-flexible, potentially deformable, and brittle-rigid regions, respectively. The layered van der Waals materials are shown as green symbols; our experimental results are shown in red. (b) Deformability factor dependence on the bandgap for the same materials as panel a. The dashed line encircled green area are materials that show exceptional plastic behavior. $\mathrm{The}$ bandgaps for $\mathrm{CrCl}_{3}$ and $\mathrm{CrI}_{3}$ nanosheets are 3.44 and $1.53 \mathrm{eV}$, respectively. ${ }^{33}$ Panel c shows a folding sequence of flat bulk crystals of $\mathrm{CrCl}_{3}$ (top strip) and CrI ${ }_{3}$ (bottom strip) into a ring-like structure (enlarged in Figure S12). The scale bars are $1 \mathrm{~mm}$.

smaller the ionic character the larger the bond energies, ${ }^{26}$ with a variation of the electronic localization function across the different Cr-halides. ${ }^{30}$ These results underline the soft nature of the chromium trihalides, which makes them extremely sensitive to small stress changes and very effective for strain modulation.

Although these results place the chromium trihalides as one of the softest $2 \mathrm{D}$ materials that have been experimentally measured so far, their breaking strength up to $10 \mathrm{~L}$ is larger than that of silicon $(\sim 2 \mathrm{GPa}){ }^{22}$ showcasing the general outstanding mechanical properties of $2 \mathrm{D}$ materials. It is also important to consider the significance of the presence of imperfections in the crystals, which affects the elastic behavior. Griffith described how the breaking strength in brittle materials (see also section 7 on the Supporting Information) is governed by defects and imperfections, ${ }^{31}$ establishing a limit of $\sigma \sim E / 9$ by experimental extrapolation. In the limit of an ideal material, mechanics are governed by its molecular tensile strengths. In both chromium trihalides, the bilayers $(\sigma \sim E / 20)$ and the multilayers $(\sigma \sim E / 10)$ follow a behavior close to this limit. These results suggest that the mechanical behavior in $\mathrm{CrX}_{3}$ thin crystals is determined by the interatomic interactions rather than defects, indicating a high crystallinity and a low density of impurities in the suspended regions. In comparison, polycrystalline classical materials like silicon ${ }^{22}$ or tungsten alloys, ${ }^{32} \sigma<E / 100$, report much lower values. ${ }^{31}$ The nonlinear elastic constitutive behavior was assumed for modeling $\mathrm{CrX}_{3}$ few layer crystals in FEM, and the derived maximum strains are close to $\sim 6-6.5 \%$ for the bilayers (see section 5 on the Supporting Information). The prospects of the combination of the exceptional flexibility and strengths with the intrinsic magnetism of atomically thin $\mathrm{CrX}_{3}$ nature hold promise for an enhanced strain-tunability in ultrathin magneto-mechanical devices. $^{33}$

Considering the remarkable flexibility of few-layer $\mathrm{CrCl}_{3}$ and $\mathrm{CrI}_{3}$, and the interlayer sliding origin of the layer-dependent Young's moduli, we investigated the plastic behavior of the two magnetic van der Waals materials in their bulk form. This property is of great relevance for future flexible devices, and it has recently been observed in bulk crystals of $\mathrm{InSe}^{25}$ The deformability factor $(\Xi)$ proposed by Wei T-R et al. can be useful as a way to frame the plastic behavior of a material, it is related to the sliding $\left(E_{\mathrm{s}}\right)$ and cleavage $\left(E_{\mathrm{c}}\right)$ energies of layered materials via

$$
\Xi=\left(E_{\mathrm{c}} / E_{\mathrm{s}}\right) \cdot(1 / E)
$$

where $\mathrm{E}$ is the volumetric Young's modulus. The magnitudes of $\Xi$ for bulk $\mathrm{CrCl}_{3}$ and $\mathrm{CrI}_{3}$ are plotted in Figure $5 \mathrm{a}$ and $5 \mathrm{~b}$ as a function of the Young modulus and bandgaps for different materials with different electronic properties (semiconducting, insulators and metals). The cleavage energies were defined as the energies to separate bilayer $\mathrm{CrX}_{3}$ systems to two monolayers (see Figure S6), the sliding energies were taken from the most energetically favored sliding path in the equilibrium state, that is, along [010] direction (Figure 3), and the Young's modulus values were extracted from the experimental data for $2 \mathrm{~L} \mathrm{CrCl}_{3}$ and $\mathrm{CrI}_{3}$. For both bulk $\mathrm{CrCl}_{3}$ and $\mathrm{CrI}_{3}$ the cleavage energies are larger than their sliding energies (see Table S3). Interestingly, the magnetic $\mathrm{CrX}_{3}$ showed one of the highest deformability factors of the $2 \mathrm{D}$ materials, even larger than that of the recently reported InSe (Figure 5). ${ }^{25}$ This outstanding capability for deformation is experimentally illustrated by macroscopically folding bulk $\mathrm{CrI}_{3}$ and $\mathrm{CrCl}_{3}$ crystals in Figure 5c. Upon further testing, $\mathrm{CrX}_{3}$ multilayered crystals could be confirmed to exhibit a superplastic behavior, which would open the door for their use in easily deformable and flexible devices that incorporate intrinsic magnetism.

\section{ASSOCIATED CONTENT}

\section{SI Supporting Information}

The Supporting Information is available free of charge at https://pubs.acs.org/doi/10.1021/acs.nanolett.0c04794.

Details on the bulk crystal growth, the fabrication of suspended atomically thin crystals, the characterization of the AFM indenters, the finite element analysis, the $a b$ initio calculations performed, and supporting results regarding the plasticity in bulk crystals of $\mathrm{CrX}_{3}$ (PDF)

\section{AUTHOR INFORMATION}

\section{Corresponding Authors}

Elton J. G. Santos - Institute for Condensed Matter Physics and Complex Systems, School of Physics and Astronomy and 
Higgs Centre for Theoretical Physics, The University of Edinburgh, EH9 3FD Edinburgh, United Kingdom; Email: esantos@ed.ac.uk

Lu Hua Li - Institute for Frontier Materials, Deakin University, Waurn Ponds, Victoria 3216, Australia; 구 orcid.org/0000-0003-2435-5220; Email: luhua.li@ deakin.edu.au

Efrén Navarro-Moratalla - Instituto de Ciencia Molecular, Universitat de Valencia, 46980 Paterna, Spain; @ orcid.org/ 0000-0002-3430-6599; Email: efren.navarro@uv.es

\section{Authors}

Fernando Cantos-Prieto - Instituto de Ciencia Molecular, Universitat de Vatencia, 46980 Paterna, Spain

Alexey Falin - Guangdong Provincial Key Laboratory of Functional Soft Condensed Matter, School of Materials and Energy, Guangdong University of Technology, Guangzhou 510006, China; Institute for Frontier Materials, Deakin University, Waurn Ponds, Victoria 3216, Australia

Martin Alliati - School of Mathematics and Physics, Queen's University Belfast, BT7 1NN Belfast, United Kingdom

Dong Qian - Department of Mechanical Engineering, The University of Texas at Dallas, Richardson, Texas 75080, United States; 10 orcid.org/0000-0001-9367-0924

Rui Zhang - Department of Mechanical Engineering, The University of Texas at Dallas, Richardson, Texas 75080, United States

Tao Tao - Guangdong Provincial Key Laboratory of Functional Soft Condensed Matter, School of Materials and Energy, Guangdong University of Technology, Guangzhou 510006, China

Matthew R. Barnett - Institute for Frontier Materials, Deakin University, Waurn Ponds, Victoria 3216, Australia

Complete contact information is available at:

https://pubs.acs.org/10.1021/acs.nanolett.0c04794

\section{Author Contributions}

${ }^{\dagger}$ F.C.-P. and A.F. contributed equally. The manuscript was written through contributions of all authors. All authors have given approval to the final version of the manuscript.

\section{Notes}

The authors declare no competing financial interest.

\section{ACKNOWLEDGMENTS}

E.J.G.S. acknowledges computational resources through CIRRUS Tier-2 HPC Service (ec131 Cirrus Project) at EPCC (http://www.cirrus.ac.uk) funded by the University of Edinburgh and EPSRC (EP/P020267/1); ARCHER UK National Supercomputing Service (http://www.archer.ac.uk) via Project d429. E.J.G.S. acknowledges the EPSRC Early Career Fellowship (EP/T021578/1) and the University of Edinburgh for funding support. E.N.M. acknowledges the European Research Council (ERC) under the Horizon 2020 research and innovation programme (ERC StG, Grant Agreement No. 803092) and to the Spanish Ministerio de Ciencia, Innovación y Universidades for financial support from the Ramon y Cajal program (Grant No. RYC2018-024736-I). F.C.P. acknowledges the same institution for the FPU program (Grant No. FPU17/01587). This work was also supported by the Spanish Unidad de Excelencia "María de Maeztu" (CEX2019-000919-M). F.C.P. and E.N.M. are particularly grateful to the microscopy service at the SCSIE (Universitat de
Valencia) and the Electron Microscopy Service at the Polytechnic University of Valencia for their technical support.

\section{REFERENCES}

(1) Brown, W. F. Theory of Magnetoelastic Effects in Ferromagnetism. J. Appl. Phys. 1965, 36 (3), 994-1000.

(2) Meydan, T.; Oduncu, H. Enhancement of Magnetorestrictive Properties of Amorphous Ribbons for a Biomedical Application. Sens. Actuators, A 1997, 59 (1), 192-196.

(3) Bieńkowski, A.; Szewczyk, R. The Possibility of Utilizing the High Permeability Magnetic Materials in Construction of Magnetoelastic Stress and Force Sensors. Sens. Actuators, A 2004, 113 (3), 270-276.

(4) Bieńkowski, A.; Szewczyk, R.; Salach, J. Industrial Application of Magnetoelastic Force and Torque Sensors. Acta Phys. Pol., A 2010, 118 (5), 1008-1009.

(5) Gong, C.; Li, L.; Li, Z.; Ji, H.; Stern, A.; Xia, Y.; Cao, T.; Bao, W.; Wang, C.; Wang, Y.; Qiu, Z. Q.; Cava, R. J.; Louie, S. G.; Xia, J.; Zhang, X. Discovery of Intrinsic Ferromagnetism in Two-Dimensional van Der Waals Crystals. Nature 2017, 546 (7657), 265-269.

(6) Huang, B.; Clark, G.; Navarro-Moratalla, E.; Klein, D. R.; Cheng, R.; Seyler, K. L.; Zhong, D.; Schmidgall, E.; McGuire, M. A.; Cobden, D. H.; Yao, W.; Xiao, D.; Jarillo-Herrero, P.; Xu, X. Layer-Dependent Ferromagnetism in a van Der Waals Crystal down to the Monolayer Limit. Nature 2017, 546 (7657), 270-273.

(7) Lee, J.-U.; Lee, S.; Ryoo, J. H.; Kang, S.; Kim, T. Y.; Kim, P.; Park, C.-H.; Park, J.-G.; Cheong, H. Ising-Type Magnetic Ordering in Atomically Thin FePS 3. Nano Lett. 2016, 16 (12), 7433-7438.

(8) Wahab, D. A.; Augustin, M.; Valero, S. M.; Kuang, W.; Jenkins, S.; Coronado, E.; Grigorieva, I. V.; Vera-Marun, I. J.; NavarroMoratalla, E.; Evans, R. F. L.; Novoselov, K. S.; Santos, E. J. G. Quantum Rescaling, Domain Metastability, and Hybrid DomainWalls in 2D CrI3Magnets. Adv. Mater. 2021, 33 (5), e2004138.

(9) Augustin, M.; Jenkins, S.; Evans, R. F. L.; Novoselov, K. S.; Santos, E. J. G. Properties and Dynamics of Meron Topological Spin Textures in the Two-Dimensional Magnet CrCl3. Nat. Commun. 2021, 12 (1), 185.

(10) Gong, C.; Zhang, X. Two-Dimensional Magnetic Crystals and Emergent Heterostructure Devices. Science 2019, 363 (6428), eaav4450.

(11) Huang, B.; Clark, G.; Klein, D. R.; MacNeill, D.; NavarroMoratalla, E.; Seyler, K. L.; Wilson, N.; McGuire, M. A.; Cobden, D. H.; Xiao, D.; et al. Electrical Control of 2D Magnetism in Bilayer CrI 3. Nat. Nanotechnol. 2018, 13 (7), 544-548.

(12) Jiang, S.; Li, L.; Wang, Z.; Mak, K. F.; Shan, J. Controlling Magnetism in 2D CrI3 by Electrostatic Doping. Nat. Nanotechnol. 2018, 13 (7), 549-553.

(13) Li, T.; Jiang, S.; Sivadas, N.; Wang, Z.; Xu, Y.; Weber, D.; Goldberger, J. E.; Watanabe, K.; Taniguchi, T.; Fennie, C. J.; et al. Pressure-Controlled Interlayer Magnetism in Atomically Thin CrI 3. Nat. Mater. 2019, 18 (12), 1303-1308.

(14) Song, T.; Fei, Z.; Yankowitz, M.; Lin, Z.; Jiang, Q.; Hwangbo, K.; Zhang, Q.; Sun, B.; Taniguchi, T.; Watanabe, K.; McGuire, M. A.; Graf, D.; Cao, T.; Chu, J.-H.; Cobden, D. H.; Dean, C. R.; Xiao, D.; $\mathrm{Xu}, \mathrm{X}$. Switching 2D Magnetic States via Pressure Tuning of Layer Stacking. Nat. Mater. 2019, 18 (12), 1298-1302.

(15) Jiang, S.; Xie, H.; Shan, J.; Mak, K. F. Exchange Magnetostriction in Two-Dimensional Antiferromagnets. Nat. Mater. 2020, 19, 1295-1299.

(16) Woltjer, H. R.; Kamerlingh Onnes, H. Further Experiments with Liquid Helium. Z. Magnetic Researches. XXVIII. Magnetisation of Anhydrous $\mathrm{CrCl}_{3}, \mathrm{COCl}_{2}$, and $\mathrm{NiCl}_{2}$ at Very Low Temperatures. Leiden Communications 1925, 173b, 544-549.

(17) Lee, C.; Wei, X.; Kysar, J. W.; Hone, J. Measurement of the Elastic Properties and Intrinsic Strength of Monolayer Graphene. Science 2008, 321 (5887), 385-388.

(18) Cost, J. R.; Janowski, K. R.; Rossi, R. C. Elastic Properties of Isotropic Graphite. Philos. Mag. 1968, 17 (148), 851-854. 
(19) Blakslee, O. L.; Proctor, D. G.; Seldin, E. J.; Spence, G. B.; Weng, T. Elastic Constants of Compression-Annealed Pyrolytic Graphite. J. Appl. Phys. 1970, 41 (8), 3373-3382.

(20) Falin, A.; Cai, Q.; Santos, E. J. G.; Scullion, D.; Qian, D.; Zhang, R.; Yang, Z.; Huang, S.; Watanabe, K.; Taniguchi, T.; Barnett, M. R.; Chen, Y.; Ruoff, R. S.; Li, L. H. Mechanical Properties of Atomically Thin Boron Nitride and the Role of Interlayer Interactions. Nat. Commun. 2017, 8, 15815.

(21) Bertolazzi, S.; Brivio, J.; Kis, A. Stretching and Breaking of Ultrathin MoS2. ACS Nano 2011, 5 (12), 9703-9709.

(22) Tsuchiya, T. Tensile Testing of Silicon Thin Films. Fatigue Fract. Eng. Mater. Struct. 2005, 28 (8), 665-674.

(23) Castellanos-Gomez, A.; van Leeuwen, R.; Buscema, M.; van der Zant, H. S. J.; Steele, G. A.; Venstra, W. J. Single-Layer $\operatorname{MoS}(2)$ Mechanical Resonators. Adv. Mater. 2013, 25 (46), 6719-6723.

(24) Zhao, Q.; Frisenda, R.; Wang, T.; Castellanos-Gomez, A. InSe: A Two-Dimensional Semiconductor with Superior Flexibility. Nanoscale 2019, 11 (20), 9845-9850.

(25) Wei, T.-R.; Jin, M.; Wang, Y.; Chen, H.; Gao, Z.; Zhao, K.; Qiu, P.; Shan, Z.; Jiang, J.; Li, R.; Chen, L.; He, J.; Shi, X. Exceptional Plasticity in the Bulk Single-Crystalline van Der Waals Semiconductor InSe. Science 2020, 369 (6503), 542-545.

(26) Dean, J. A. Lange's Handbook of Chemistry; McGraw-Hill, Inc.: New York, 1999.

(27) Castellanos-Gomez, A.; Poot, M.; Amor-Amorós, A.; Steele, G. A.; van der Zant, H. S. J.; Agraï, N.; Rubio-Bollinger, G. Mechanical Properties of Freely Suspended Atomically Thin Dielectric Layers of Mica. Nano Res. 2012, 5 (8), 550-557.

(28) Liu, K.; Yan, Q.; Chen, M.; Fan, W.; Sun, Y.; Suh, J.; Fu, D.; Lee, S.; Zhou, J.; Tongay, S.; Ji, J.; Neaton, J. B.; Wu, J. Elastic Properties of Chemical-Vapor-Deposited Monolayer MoS2, WS2, and Their Bilayer Heterostructures. Nano Lett. 2014, 14 (9), 5097-5103.

(29) Costescu, B. I.; Baldus, I. B.; Gräter, F. Graphene Mechanics: I. Efficient First Principles Based Morse Potential. Phys. Chem. Chem. Phys. 2014, 16 (24), 12591-12598.

(30) Kartsev, A.; Augustin, M.; Evans, R. F. L.; Novoselov, K. S.; Santos, E. J. G. Biquadratic Exchange Interactions in Two-Dimensional Magnets. npj Computational Materials 2020, 6 (1), 150.

(31) Griffith, A. A.; Taylor, G. I., VI. The Phenomena of Rupture and Flow in Solids. Philosophical Transactions of the Royal Society of London 1921, 221 (582-593), 163-198.

(32) Gong, X.; Fan, J.; Ding, F. Tensile Mechanical Properties and Fracture Behavior of Tungsten Heavy Alloys at $25-1100^{\circ}$ C. Mater. Sci. Eng., A 2015, 646, 315-321.

(33) Zhang, W.-B.; Qu, Q.; Zhu, P.; Lam, C.-H. Robust Intrinsic Ferromagnetism and Half Semiconductivity in Stable Two-Dimensional Single-Layer Chromium Trihalides. J. Mater. Chem. C 2015, 3 (48), 12457-12468. 